\title{
Crecimiento y supervivencia de larvas de Rhamdia quelen criadas en estanques luego de diferentes períodos de larvicultura intensiva
}

\author{
Agüero, C.H.; Hernández, D.R.; Roux, J.P.; Sánchez, S.; Santinón, J.J. \\ Instituto de Ictiología del Nordeste, Facultad de Ciencias Veterinarias, Universidad Nacional del Nordeste, \\ Sargento Cabral 2139, Corrientes (3400), Argentina. Tel 0379-4425753 interno 171. \\ E-mail: carloshernanaguero3@hotmail.com.ar
}

\begin{abstract}
Resumen
Agüero, C.H.; Hernández, D.R.; Roux, J.P.; Sánchez, S.; Santinón, J.J.: Crecimiento y supervivencia de larvas de Rhamdia quelen criadas en estanques luego de diferentes períodos de larvicultura intensiva. Rev. vet. 25: 1, 34-39, 2014. El objetivo del presente estudio fue evaluar el efecto de la duración de la larvicultura intensiva bajo condiciones controladas sobre el crecimiento y supervivencia de larvas de Rhamdia quelen. Se realizaron 4 tratamientos experimentales: $\left(\mathrm{L}_{0}\right)$ donde las larvas fueron colocadas directamente a estanques al inicio de la alimentación exógena, o mantenidas inicialmente en laboratorio hasta los $5\left(\mathrm{~L}_{1}\right)$, $10\left(\mathrm{~L}_{2}\right)$ y $15\left(\mathrm{~L}_{3}\right)$ días posteriores a la eclosión (dpe) antes de ser transferidas a estanques. El ensayo tuvo una duración de 45 días. Al final del ciclo completo (45 dpe), $\mathrm{L}_{0}$ y $\mathrm{L}_{1}$ presentaron los mayores valores de peso (12,8 y 10,4 g, respectivamente). A su vez, $\mathrm{L}_{0}$ presentó el porcentaje de supervivencia más bajo $(0,7 \%, \mathrm{p}<0,05)$, mientras que $\mathrm{L}_{2}$ presentó valores de supervivencia intermedios, logrando la mayor biomasa final $(\mathrm{p}<0,05)$. El análisis de la etapa de larvicultura en laboratorio permitió detectar una relación negativa en la supervivencia de las larvas en función de la permanencia de las mismas en este sistema (73,6; 58,9 y 44\% para $\mathrm{L}_{1}, \mathrm{~L}_{2} \mathrm{y} \mathrm{L}_{3}$, respectivamente), mientras que esta relación fue positiva una vez que las larvas se encontraron en los estanques $\left(5,25 ; 32,58\right.$ y $49,88 \%$ para $\mathrm{L}_{1}, \mathrm{~L}_{2}$ y $\mathrm{L}_{3}$, respectivamente). Bajo las condiciones experimentales del presente trabajo, el momento más recomendable para el pasaje de larvas a estanques externos sería a los 10 dpe, ya que se obtienen valores de supervivencia aceptables y pesos adecuados para el inicio de la etapa de recría.
\end{abstract}

Palabras clave: pez, Rhamdia quelen, larvicultura, crecimiento, supervivencia.

\begin{abstract}
Agüero, C.H.; Hernández, D.R.; Roux, J.P.; Sánchez, S.; Santinón, J.J.: Growth and survival of Rhamdia quelen larvae reared in ponds after different periods of intensive larviculture. Rev. vet. 25: 1, 34-39, 2014. The aim of this study was to evaluate the effect of the duration of intensive larviculture on growth and survival of Rhamdia quelen larvae under experimental conditions. A total of four experimental treatments were performed, consisting of a semi-intensive larviculture $\left(\mathrm{L}_{0}\right)$ in ponds at the start of exogenous feeding, or intensive larviculture for $5\left(\mathrm{~L}_{1}\right), 10\left(\mathrm{~L}_{2}\right)$ and $15\left(\mathrm{~L}_{3}\right)$ days post-hatching $(\mathrm{dph})$ before transfer to ponds. The experience lasted for 45 days. At the end of the complete cycle ( $45 \mathrm{dph}), \mathrm{L}_{0}$ and $\mathrm{L}_{1}$ showed the highest weight values (12.8 and $10.4 \mathrm{~g}$, respectively). In turn, $\mathrm{L}_{0}$ presented lower survival rate $(0.7 \%, p<0.05)$, while $L_{2}$ showed intermediate survival values, obtaining the highest final biomass $(p<0.05)$. The analysis of the intensive larviculture stage revealed a negative relationship on the survival rate of larvae based on their permanence in the system (73.6, 58.9 and $44 \%$ for $\mathrm{L}_{1}, \mathrm{~L}_{2}$ and $\mathrm{L}_{3}$, respectively), whereas this relationship was positive once the larvae were placed in ponds $\left(5.25,32.58\right.$ and $49.88 \%$ for $\mathrm{L}_{1}, \mathrm{~L}_{2}$ and $\mathrm{L}_{3}$, respectively). The most suitable time for larvae relocation in external ponds under the experimental conditions would be at $10 \mathrm{dph}$, since acceptable survival rates and appropriate weights are obtained for the beginning of fingerling rearing stage.-
\end{abstract}

Key words: fish, Rhamdia quelen, larviculture, growth, survival. 


\section{INTRODUCCIÓN}

La etapa de larvicultura, de la cual depende la producción a gran escala de ejemplares juveniles ${ }^{12}$, es considerada una de las fases más difíciles de la acuicultura ${ }^{19}$. Este período presenta diversos inconvenientes, relacionados generalmente a la falta de conocimiento biológico de las especies a cultivar.

En Sudamérica, el sistema de larvicultura comúnmente utilizado es el semi-intensivo, en el cual una vez que las larvas inician la alimentación exógena son transferidas a estanques excavados o jaulas suspendidas donde se alimentan de plancton ${ }^{13,23}$. En este sistema, la supervivencia de las larvas depende tanto de factores intrínsecos como extrínsecos. Dentro de los primeros se encuentran el grado de desarrollo del tubo digestivo, sistema sensorial y aparato locomotor. Dentro de los factores extrínsecos se destacan las condiciones ambientales, principalmente la temperatura del agua, densidad de stock, tipo de alimento y presencia de depredadores ${ }^{10}$. En general, este sistema se caracteriza por presentar bajos porcentajes de supervivencia (de 0,1 a $30 \%)^{7}$.

Otro método que en la actualidad está siendo ampliamente utilizado es el sistema intensivo de larvicultura, donde las larvas son mantenidas bajo condiciones controladas y alimentadas con alimento vivo (rotíferos, copépodos y Artemia sp.), microdietas (MD) o una co-alimentación (alimento vivo $+\mathrm{MD}$ ) hasta la etapa de juveniles. Este sistema permite un desarrollo más avanzado de las larvas antes de ser tansferidas a estanques, lo cual mejora las chances de supervivencia de las mismas ${ }^{8,17}$. En las últimas dos décadas, varios estudios se enfocaron en maximizar la productividad en sistemas intensivos de larvicultura, alcanzando, en algunos casos, niveles de supervivencia superiores al $90 \%{ }^{4,5}$. Sin embargo, esta metodología de trabajo requiere instalaciones adecuadas para mantener a los peces a densidades elevadas, aumentando considerablemente los costos de producción ${ }^{14}$.

Una práctica más reciente se basa en una combinación de estos sistemas de larvicultura. Consiste en reducir el tiempo de permanencia de las larvas en laboratorio hasta garantizar un apropiado desarrollo de las mismas antes de ser transferidas a los estanques externos. Esto disminuye la elevada mortalidad causada por el cambio brusco de ambiente y tipo de alimento, así como la posibilidad de ser depredadas ${ }^{2,13,15}$.

Diversos trabajos fueron orientados a evaluar el desempeño productivo de larvas de $R$. quelen dentro de los primeros días de vida bajo condiciones controladas $2,4,5$, aunque resultan escasos los estudios dedicados a determinar el momento adecuado para transferir las larvas a estanques o jaulas de cría o recría con el objetivo de disminuir el tiempo de larvicultura en laboratorio. En este contexto, el objetivo del presente estudio fue evaluar el momento más recomendable para la transferencia de larvas de $R$. quelen desde un sistema controlado de larvicultura en laboratorio a estanques de cría externos.

\section{MATERIAL Y MÉTODOS}

La experiencia se llevó a cabo en las instalaciones de piscicultura experimental del Instituto de Ictiología del Nordeste (INICNE) de la Facultad de Ciencias Veterinarias-UNNE, Corrientes, Argentina.

Diseño del bioensayo. Las larvas de $R$. quelen fueron obtenidas mediante cruzamientos entre ejemplares capturados en ambientes naturales, previa inducción hormonal con extracto de hipófisis de Prochilodus lineatus. Luego del desove y fertilización, los huevos fueron mantenidos en incubadoras de 50 litros de capacidad hasta la eclosión. Fueron evaluados cuatro momentos de transferencia de las larvas a estanques; $\mathrm{L}_{0}$, $\mathrm{L}_{1}, \mathrm{~L}_{2}$ y $\mathrm{L}_{3}$, correspondientes a peces de $2,5,10$ y 15 días post-eclosión (dpe), respectivamente. Las larvas $\mathrm{L}_{0}$ (de dos días de edad e iniciadas en su alimentación exógena), fueron trasladadas directamente a estanques externos bajo un sistema de larvicultura semi-intensiva. Para los tratamientos $\mathrm{L}_{1}, \mathrm{~L}_{2}$ y $\mathrm{L}_{3}$, se realizaron transferencias desde larvicultura intensiva en laboratorio a estanques en diferentes períodos de tiempo $(5,10$ y 15 dpe, respectivamente). Los tratamientos experimentales se designaron de acuerdo al período de larvicultura intensiva recibida (Figura 1).

Larvicultura en laboratorio. Lotes de 750 larvas fueron repartidos en 9 acuarios (3 réplicas por tratamiento) conteniendo 25 litros de agua de perforación y aireación forzada (densidad de 30 ind/l), con una sustitución de agua a razón de 0,4 1/min durante 12 horas al día para la eliminación de residuos. Hasta el momento de la transferencia a estanques, las larvas fueron alimentadas con ración balanceada elaborada con levadu-

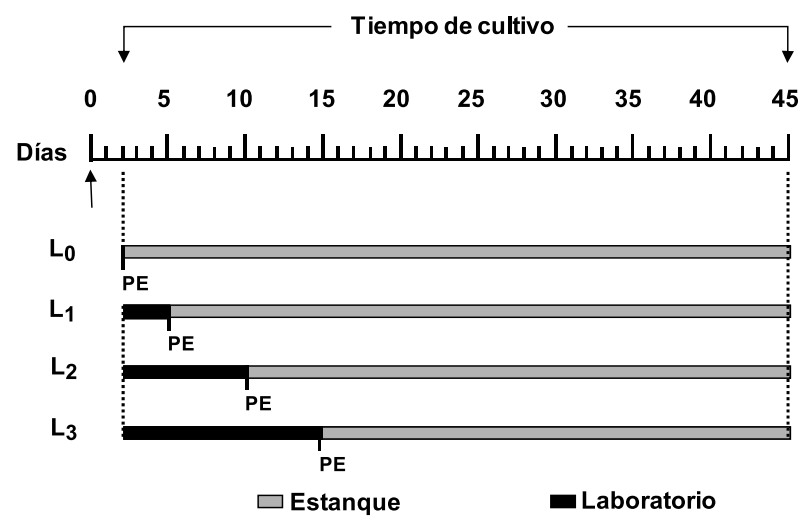

Figura 1. Esquema de los diferentes tratamientos experimentales utilizados en la larvicultura de $R$. quelen. El día 0 (flecha) corresponde al momento de la eclosión de las larvas. Línea punteada indica el inicio de la alimentación exógena ( 2 dpe). PE: pasaje a estanques externos. 
ra de pan $57 \%$, huevo de pescado $35 \%$, lecitina de soja $2 \%$ y núcleo vitamínico-mineral $6 \%$. La pasta obtenida fue pasada por un picador para formar pellets de $4 \mathrm{~mm}$ de diámetro, los que se secaron en estufa a $40^{\circ} \mathrm{C}$ durante $48 \mathrm{~h}$. La ración fue molida y tamizada de acuerdo con el tamaño de apertura bucal de las larvas con granulometrías entre $100-200 \mu \mathrm{m}$ y $250-450 \mu \mathrm{m}$ para la primera y segunda semana de vida, respectivamente. Todos los grupos de larvas fueron alimentados hasta saciedad cuatro veces al día $(8,12,16$ y 20 h), durante todo el período experimental.

Larvicultura semi-intensiva. Tres lotes de 400 larvas ( 2 dpe) fueron transferidos directamente a estanques de mampostería de $4 \mathrm{~m}^{3}$ y $50 \mathrm{~cm}$ de profundidad ( $2 \mathrm{~m}^{3}$ de capacidad) conformando el grupo $\mathrm{L}_{0}$ (densidad de $200 \mathrm{ind} / \mathrm{m}^{3}$ ). Esta metodología se repitió en cada etapa de transferencia de larvas a estanques (tratamientos $\mathrm{L}_{1}, \mathrm{~L}_{2} \mathrm{y} \mathrm{L}_{3}$ ). Una semana antes del pasaje de las larvas, los estanques fueron fertilizados con alfalfa a razón de $300 \mathrm{~g} / \mathrm{m}^{2}$. Durante la cría en estanques, los peces fueron alimentados hasta saciedad cuatro veces al día con ración balanceada comercial conteniendo $28 \%$ de proteínas.

Variables de calidad de agua. La temperatura del agua $\left({ }^{\circ} \mathrm{C}\right)$ y el oxígeno disuelto (OD) se registraron diariamente con oxímetro digital $\mathrm{YSI}^{\circledR}$. La conductividad (CO) y $\mathrm{pH}$ se registraron con frecuencia semanal con instrumentos HANNA ${ }^{\circledR}$. Los parámetros de calidad de agua obtenidos en laboratorio fueron: temperatura del agua $25,9^{\circ} \mathrm{C}$; $\mathrm{pH} 6,4$; OD 7,4 mg/l y $97,2 \mu \mathrm{S} / \mathrm{cm}$ de $\mathrm{CO}$, mientras que en los estanques el promedio fue de $24,4^{\circ} \mathrm{C} ; \mathrm{pH} 8$; OD $10,3 \mathrm{mg} / \mathrm{l}$ y $138,7 \mu \mathrm{S} / \mathrm{cm}$ de $\mathrm{CO}$.

Registro de datos y análisis estadístico. Al final de cada período de larvicultura en laboratorio $(5,10$ y 15 dpe), así como al final de la experiencia (45 días de du- ración), las variables de peso medio final (PM), tasa de crecimiento específica (TCE), porcentaje de supervivencia $(\% \mathrm{~S})$ y biomasa final $(\mathrm{BF})$ fueron calculadas de la siguiente manera: $\mathrm{PM}(\mathrm{g})=\mathrm{P}_{t} / N$, donde $\mathrm{P}_{t}$ es el peso total (g) por cada réplica y $N=$ número de individuos sobrevivientes; TCE $=100\left[\left(\ln P_{f}-\ln P_{i}\right) /(\mathrm{T})\right]$, donde $\ln$ es el logaritmo natural de $P_{f}=$ peso medio final y $P_{i}=$ peso medio inicial expresado en $\mathrm{g}$ de un período determinado (T); $\mathrm{S}(\%)=100\left(N / N_{i}\right)$, donde $N_{i}=$ número inicial de peces; $\mathrm{BF}=\mathrm{PM}(N)$. Los resultados obtenidos se analizaron mediante análisis de la varianza a una vía, según un diseño completamente aleatorizado. Los valores medios resultantes de las mediciones de crecimiento se analizaron mediante regresión. Las comparaciones de medias de tratamientos a posteriori se realizaron mediante el test de Tuckey $(p<0,05)$. El software utilizado para el análisis de los datos fue Statistica for Windows, versión 6.0.

\section{RESULTADOS}

Larvicultura en laboratorio. Los resultados obtenidos evidenciaron un incremento gradual para la variable PM, alcanzando valores de 1,$68 ; 4,81$ y 15,29 mg para los tratamientos $\mathrm{L}_{1}, \mathrm{~L}_{2} \mathrm{y} \mathrm{L}_{3}$, respectivamente (Figura 2-a). Por su parte, el \%S mostró una disminución ante el aumento de la permanencia de las larvas en laboratorio, presentando valores de 73,6, 58,9 y 44\% para $\mathrm{L}_{1}, \mathrm{~L}_{2}$ y $\mathrm{L}_{3}$, respectivamente (Figura 2-a). Con respecto a la TCE, no se observaron diferencias entre los distintos tratamientos experimentales $(p>0,05)$, pero si se comprobó una relación significativa entre este valor y el \%S (Figura 2-b).

Larvicultura semi-intensiva. Al final de los 45 días de ensayo, el \%S evidenció diferencias significativas para los grupos transferidos a estanques, presentando valores superiores para los tratamientos $\mathrm{L}_{2} \mathrm{y} \mathrm{L}_{3}$

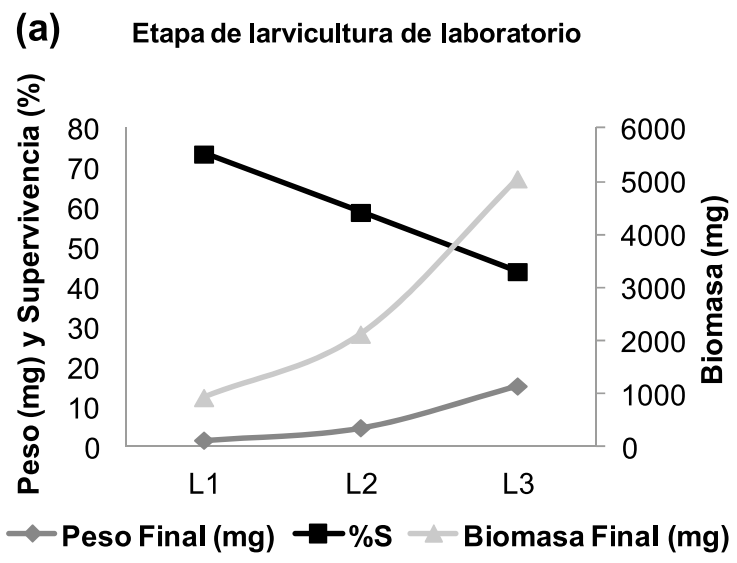

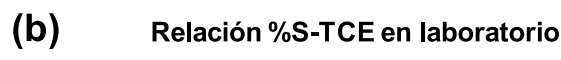

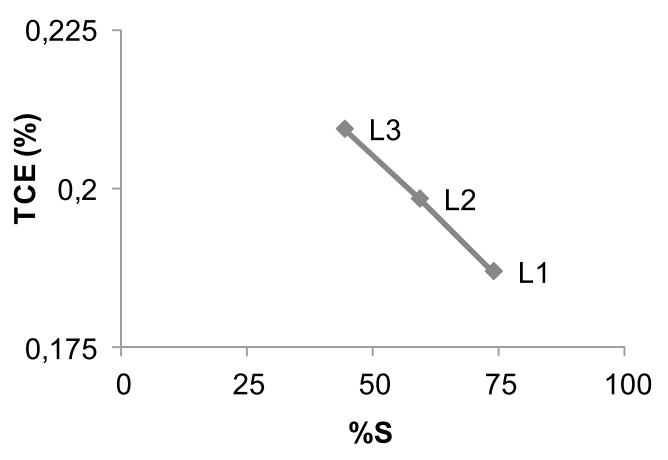

Figura 2. a) Valores promedios de peso medio (PM), porcentaje de supervivencia (\%S) y biomasa final (BF) obtenidos en larvas de $R$. quelen criadas bajo un sistema de larvicultura intensiva en laboratorio. Parámetros obtenidos desde el inicio del ensayo hasta los 15 días de vida $(5,10$ y $15 \mathrm{dpe})$. b) Relación entre \% y tasa de crecimiento especifico (TCE) en larvas de $R$. quelen criadas bajo un sistema de larvicultura intensiva en laboratorio. 
(a) Etapa de larvicultura semi-intensiva en estanques

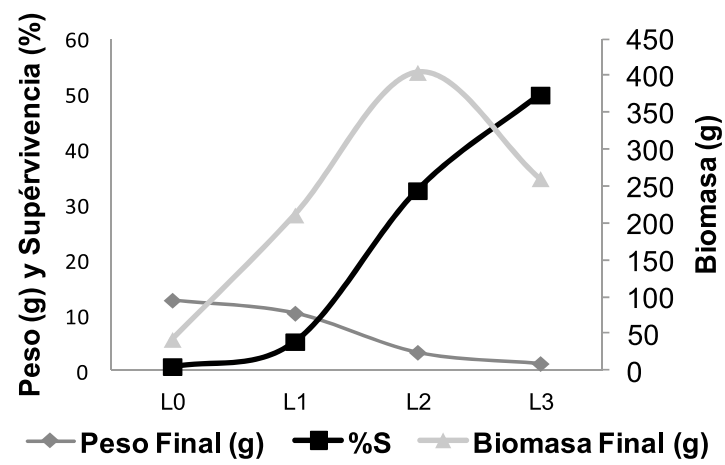

(b) Relación \%S-TCE en estanques

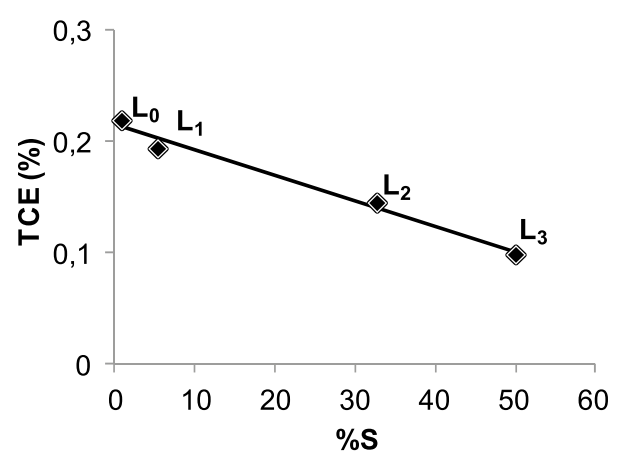

Figura 3. a) Valores promedios de peso medio PM, porcentaje de supervivencia $(\% \mathrm{~S})$ y biomasa final $(\mathrm{BF})$ en larvas de $R$. quelen criadas bajo un sistema de larvicultura semi-intensivo. b) Relación entre \% $\mathrm{S}$ y tasa de crecimiento especifico (TCE) en larvas de $R$. quelen criadas bajo un sistema de larvicultura semi-intensivo.

$(\mathrm{p}<0,05)$ con 32,58 y $49,88 \%$ respectivamente, al tiempo que los tratamientos $\mathrm{L}_{0} \mathrm{y} \mathrm{L}_{1}$ presentaron los menores porcentajes, alcanzando valores de 0,75 y $5,25 \%$, respectivamente (Figura 3-a).

Para la variable de PM, los tratamientos $\mathrm{L}_{0}(12,8 \mathrm{~g})$ y $\mathrm{L}_{1}(10,4 \mathrm{~g})$, no presentaron diferencias significativas entre sí $(\mathrm{p}>0,05)$, resultando mayores a los valores de

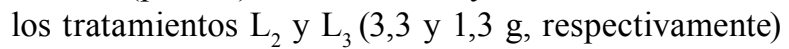
(Figura 3-a). Con respecto a la BF, el tratamiento $\mathrm{L}_{2}$ alcanzó valores superiores, diferenciándose estadísticamente del resto $(\mathrm{p}<0,05)$ (Figura 3-a). Además, se observó una relación negativa entre el \% y la TCE, similar a lo encontrado en la etapa de laboratorio (Figura 3-b).

Al analizar la experiencia (45 dpe) considerando las etapas intensiva y semi-intensiva de larvicultura, se observó que los mejores resultados correspondieron a $\mathrm{L}_{2}$, tratamiento que presentó elevado $\% \mathrm{~S}$, similares a $\mathrm{L}_{3}$, pero que al presentar un PM final superior $\left(\mathrm{L}_{2}=63,72\right.$ g) resultó en una mayor BF del sistema $\left(\mathrm{L}_{0}=7,21 \mathrm{~g} ; \mathrm{L}_{1}=\right.$ 40,52 g y $_{3}=28,57$ g) (Figura 4). El ajuste polinómico de esos resultados indica que la $\mathrm{BF}$ producida podría ser máxima alrededor de los 8 dpe. (Figura 4).

\section{DISCUSIÓN}

En el presente trabajo las larvas mantenidas en laboratorio presentaron valores de PM mayores a medida que las larvas permanecieron bajo este sistema aunque la TCE fue similar para los 5, 10 y 15 dpe. Diferentes ensayos de larvicultura realizados en $R$. quelen a densidades similares a la utilizada en el presente estudio demostraron que existen pocas diferencias entre las larvas en cuanto a parámetros de crecimiento dentro de los primeros 10 días de vida para diferentes tipos de alimentos probados ${ }^{4,21}$.

$\mathrm{El} \% \mathrm{~S}$ mostró una disminución en relación al tiempo en que las larvas permanecieron bajo este sistema. Trabajos realizados en larvicultura intensiva de
R. quelen, revelaron una notoria disminución en el $\% \mathrm{~S}$ a medida que las larvas permanecieron en laboratorio, independientemente del tipo de alimento otorgado ${ }^{4,21}$. Además, se obtuvieron mejores PM finales en relación al tiempo de permanencia bajo ese sistema. Coincidentemente, en este estudio se observó un aumento en el PM, demostrando que el mejor desempeño puede asociarse a la densidad de cultivo más que al tiempo de permanencia en laboratorio. Contrariamente, en larvas de pacú (Piaractus mesopotamicus) se observó escaso crecimiento al utilizar una densidad de 20 larvas/1, comparadas con densidades superiores donde se obtuvieron los mejores resultados ${ }^{22}$.

En este estudio, y al igual que lo observado por otros investigadores $1,13,18$, se pudo constatar que el sistema semi-intensivo de larvicultura presenta bajo porcentaje de supervivencia, haciéndose muy difícil la producción a gran escala con este tipo de sistema ${ }^{12}$.

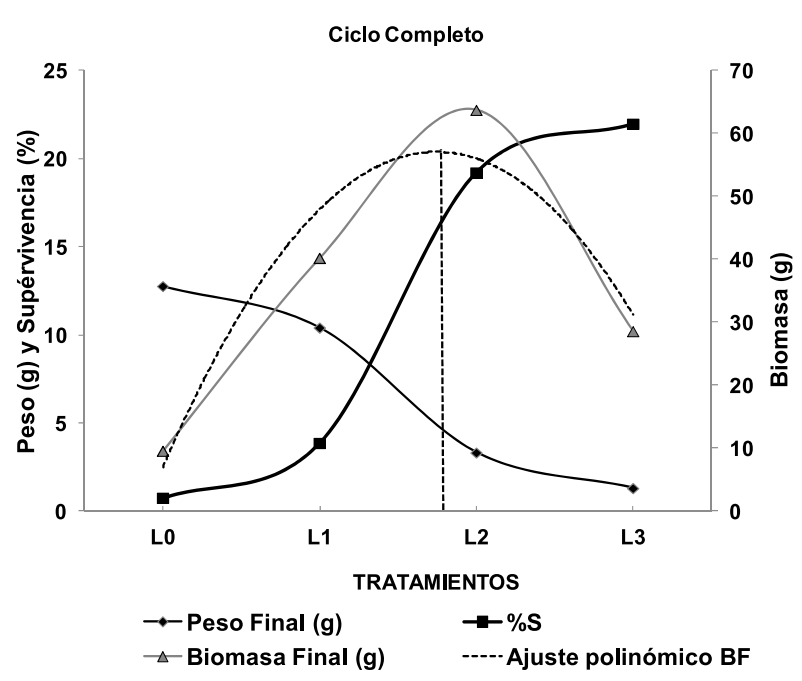

Figura 4. Valores de peso medio final (PM), porcentaje de supervivencia $(\% \mathrm{~S})$ y biomasa final (BF) obtenidos en larvas de $R$. quelen durante el ciclo completo. 
Esto posiblemente se debe a la falta de adaptación a las condiciones ambientales, a una incapacidad de las larvas en reconocer y capturar el alimento al inicio de la alimentación exógena, a posibles enfermedades transmitidas por otros organismos acuáticos o ser depredados por insectos y sus larvas, representando las amenazas más grave en este estadio ${ }^{9}$.

Si bien en $\mathrm{L}_{0}$ y $\mathrm{L}_{1}$ se observó la tasa de supervivencia más baja, estos grupos presentaron los PM más altos que el resto, posiblemente debido a un menor estrés por densidad ${ }^{3} \mathrm{y}$ una mayor disponibilidad de alimento natural en el estanque ${ }^{16}$. Algunos autores sugieren que el zooplancton es fundamental como alimento para un buen crecimiento de las larvas, principalmente dentro de los primeros 30 días de vida, dado que el zooplancton posee altos niveles de proteínas de excelente calidad $^{11,16}$.

Se ha demostrado ampliamente que modificando la densidad de peces en un estanque pueden obtenerse resultados muy dispares en lo que respecta al PM individual, así como a la biomasa total producida por unidad de superficie, variables que en general se correlacionan negativamente ${ }^{20}$. El sistema semi-intensivo produce animales de tallas muy heterogéneas, lo cual dificulta la categorización para la etapa de recría ${ }^{13}$, de cualquier modo, este sistema sigue siendo uno de los más utilizados ${ }^{14}$.

Al finalizar el presente estudio las larvas transferidas entre los 10 o 15 dpe mostraron los mejores parámetros productivos, sin embargo el ajuste polinómico detectó que para obtener el máximo rendimiento de BF, el mejor momento para transferir las larvas a estanques debería realizarse a los 8 dpe, ya que se puede obtener mejor porcentaje de supervivencia, lotes homogéneos y peso acorde para iniciar la etapa de recría ${ }^{2}$. Además, al disminuir la duración de la larvicultura en laboratorio, los costos operativos disminuyen, así como la posibilidad de aparición de patologías asociadas al incorrecto desarrollo esquelético en los sistemas de larvicultura intensiva ${ }^{6}$. Estudios realizados en larvas de Salminus brasiliensis mostraron que cuando fueron alimentadas en laboratorio durante más tiempo (4 y 6 días) presentaron $\% \mathrm{~S}$ y crecimiento mayores que aquellas mantenidas entre cero y dos días ${ }^{18}$. De igual manera, en larvas de pacú ( $P$. mesopotamicus) se obtuvieron altos $\% \mathrm{~S}$ luego de una larvicultura de 9 días en sistema intensivo bajo condiciones controladas de laboratorio ${ }^{13}$.

Los resultados obtenidos bajo las condiciones experimentales del presente estudio demuestran que es posible obtener porcentajes aceptables de supervivencia en larvas de $R$. quelen al ser mantenidas hasta los 10 dpe en condiciones controladas de laboratorio, siendo además el mejor momento para la transferencia a estanques.

Adenda. Los procedimientos adoptados con los animales experimentales fueron aprobados por el comité de Bioética y Seguridad de la Facultad de Ciencias Veterinarias UNNE (Protocolo 0001/14_2011_02827).

\section{REFERENCIAS}

1. Atencio V, Zaniboni E, Pardo S, Arias A. 2003. Influência da primeira alimentação na larvicultura e alevinagem do yamú Brycon siebenthalae (Characidae). Acta SciMaringá 25: 61-72.

2. Baldisserotto B, Radünz J. 2004. Criação de jundiá, Ed. UFSM, Santa María (Brasil), 232 p.

3. Barcellos LJ, Kreutz LC, Quevedo RM, Fioreze I, Cericato L, Benck A, Fagundes M, Conrad J, Krammer R, Bruschi A, Ritter F. 2004. Nursery rearing of jundiá, Rhamdia quelen (Quoy \& Gaimard) in cages: cage type, stocking density and stress response to confinement. Aquaculture 232: 383-394.

4. Behr ER, Tronco AP, Radünz J. 2000. Ação do tempo e da forma de suplementação alimentar com Artemia franciscana sobre a sobrevivência e o crescimento de larvas de jundiá. Ciênc Rural 30: 503-507.

5. Cardoso AP, Radünz J, Dos Santos T, Knopker A, Lazzari R. 2004. Criação de larvas de jundiá (Rhamdia quelen) alimentadas com rações granuladas contendo fígado ou hidrolisados. Acta Sci Maringá 26: 457-462.

6. Cahu C, Zambonino J, Takeuchi T. 2003. Nutritional components affecting skeletal development in fish larvae. Aquaculture 227: 245-258.

7. Chabalin E, Senhorini JA, Ferraz JA. 1989. Estimativa de custo de produção de larvas e alevinos. Boletim Técnico do CEPTA 2: 61-74.

8. Civera R, Alvarez CA, Moyano FJ. 2004. Nutrición y alimentación de larvas de peces marinos. Memorias VII Simp Intern Nutr Acuícola, Hermosillo (Sonora, México), p. 8-94.

9. FAO. 1981. Propagación artificial de peces de aguas templadas: manual para extensionistas (Woynarovich E, Horváth L.). Documento Técnico de Pesca. 201, 187 p.

10. Faria ACA, Hayashi C, Soares CM. 2001. Predação de larvas de pacu (Piaractus mesopotamicus) por copépodos ciclopóides (Mesocyclops lengisetus, Thiébaud) em diferentes densidades e ambientes e com diferentes contrastes visuais. Acta Sci Maringá 23: 497-502.

11. Furuya VR, Hayashi C, Furuya WM, Soares CM, Galdiol EM. 1999. Influência de plâncton, dieta artificial e sua combinação sobre o crescimento e sobrevivência de larvas de curimbatá (Prochilodus lineatus). Acta Sci Maringá 21: 699-703.

12. García A, Huisman EA, Sorgeloos P, Verreth J. 2001. Evaluation of protein quality in microbound started diets made with decapsulated cysts of Artemia and fish meal for fish larvae. J World Aquacult Soc 32: 317-329.

13. Jomori RK, Carneiro DJ, Malheiros EB, Portella MC. 2003. Growth and survival of pacu Piaractus mesopotamicus (Holmberg, 1887) juveniles reared in ponds or at different initial larviculture periods indoors. Aquaculture 221: 277-287.

14. Jomori RK, Carneiro DJ, Espagnoli MI, Portella MC. 2005. Economic evaluation of Piaractus mesopotamicus juvenile production in different rearing systems. Aquaculture 243: 175-183. 
15. Koumoundouros G, Carrillo J, Divanach P, Kentouri M. 2004. The rearing of common dentex Dentex dentex (L.) during the hatchery and on-growing phases. Aquaculture 240: 165-173.

16. Kubitza F. 1998. Nutrição e alimentação dos peixes cultivados, Ed. Projeto Pacu/. Agropeixe, Campo Grande (Brasil), p.108.

17. Lazo J. 2000. Conocimiento actual y nuevas perspectivas en el desarrollo de dietas para larvas de peces marinos. Memorias V Simp Intern Nutr Acuícola, Mérida (Yucatán, México), p. 300-312.

18. Mai MG, Zaniboni E. 2005. Efeito da idade de estocagem em tanques externos no desempenho da larvicultura do dourado Salminus brasiliensis (Osteichthyes, Characidae). Acta Sci Maringá 27: 287-296.

19. NRC (National Research Council). 1993. Nutrient requirements of fish, National Academy Press, Washington (USA), $114 \mathrm{p}$.
20. Padua DM, Silva PC, Pádua JT, Fernández CM, Andrade ML, Urbinati EC. 1997. Efeito da densidade de lotação e renovação da água no desenvolvimento do pacu, Piaractus mesopotamicus. Anais das Escolas de Agronomia e Veterinária da UFG. On line: acervo.ufvjm.edu. br:8080/jspui/.../BIOFILTROS.pdf $\rceil$

21. Piaia R, Uliana O, Felipetto J, Radünz J. 1997. Alimentação de larvas de jundiá (Rhamdia quelen), com dietas artificiais. Ciênc Nat 19: 119-131.

22. Pinto ML, Castagnolli N. 1984. Desenvolvimento inicial do pacu Colossoma mitrei (Berg, 1895). Anais do Simposio Brasileiro de Aqüicultura 3: 523-535.

23. Senhorini JÁ, Fontes NA, Lucas AF, Santos JS. 1991. Larvicultura do pacu Piaractus mesopotamicus Holmberg, 1887 (Pisces Characidae) em viveiros com e sem organofosforado (Folidol 60\%). Boletim Técnico do CEPTA 4: 1122 .

\section{Revista Veterinaria obtuvo el máximo nivel de categorización del CAICYT-CONICET}

Tras el pertinente proceso de evaluación según criterios de calidad editorial, en setiembre de 2005 CAICYT-CONICET ha clasificado a nuestra publicación con Categoría 1 (nivel superior de excelencia), con lo cual pasa a integrar el Catálogo Latindex (folio 14022). La Dirección de Revista veterinaria agradece a quienes colaboraron para obtener tan importante distinción. Ver: http://www.latindex. unam.mx/busquedas/catalogotitulo.html 